\title{
ANÁLISE DA PERCEPÇÃO DOS USUÁRIOS SOBRE A UTILIZAÇÃO DE UM SISTEMA DE INFORMAÇÃO GEOGRÁFICA EM UMA EMPRESA DO SETOR DE MINERAÇÃO
}

\author{
Gleidison Mendes Martins \\ Facci/Funcesi \\ gleidisonmm@gmail.com \\ Rafael Diego Barbosa \\ Facci/Funcesi \\ dba.rafael@gmail.com
}

Fabricio Roulin Bittencout

Facci/Funcesi

fabriciorb@gmail.com

Patrícia Carla de Brito Neves
Facci/Funcesi
patriciacb.neves@gmail.com

Thaís Cotta Barbosa da Silva

Facci/Funcesi

thais_cotta@yahoo.com.br

\begin{abstract}
Resumo
Os Sistemas de Informações Geográficas (SIG) possuem funcionalidades que permitem visualizar, elaborar, gerenciar e analisar dados geográficos para o estudo de cenários. Nesse contexto insere-se o objetivo desta pesquisa cuja proposta foi analisar a percepção dos usuários de uma empresa do ramo de mineração acerca da qualidade e do uso de um SIG. Para a realização deste estudo, utilizou-se uma abordagem qualitativa do tipo descritiva e, como método, pesquisa de campo. O universo foi composto pelas organizações atuantes que utilizam o SIG região de Itabira/MG. A amostra compreendeu oito colaboradores de uma organização que são usuários de um SIG e o critério de amostragem escolhido foi não probabilística por acessibilidade. A coleta de dados deu-se por meio de entrevistas semiestruturadas e o tratamento dos dados através da análise de conteúdo. Evidenciou-se, de acordo com os questionários, que o software Arcview possui uma integração amigável com os demais softwares da empresa e que treinamentos são fundamentais para os usuários. As informações devem estar sempre atualizadas para que não haja o risco em se trabalhar com dados obsoletos, garantindo que o banco de dados esteja sempre atualizado, confiável e centralizado. As características positivas do sistema foram descritas pelos usuários, que apontaram várias e consideraram como vitais para a evolução da utilização e eficiência do software e seus resultados. As características negativas do sistema foram descritas pelos usuários, que também listaram alguns pontos críticos e que influenciam nos resultados gerados pela utilização do SIG. De acordo com o objetivo geral desta monografia, que foi de analisar a percepção dos usuários de uma empresa de mineração acerca do uso de um SIG, foi possível concluir que os usuários conhecem o SIG da empresa de mineração pesquisada e o consideram como uma ferramenta essencial para o ambiente de processos no qual está empregado. Comprovou-se que o SIG tem inúmeras vantagens, como base de dados confiável e íntegra, que deixam clara sua importância no ambiente estudado e sua contribuição para que
\end{abstract}


as decisões e ações tomadas possam ser embasadas em informações confiáveis e sólidas.

Palavras-chaves: Sistemas de Informações Geográficas. Qualidade. Empresa de mineração.

\begin{abstract}
The Geographic Information Systems (GIS) have features that allow you to view, prepare, manage and analyze spatial data for the study of scenarios. This scenario is it the objective of this research was to analyze the proposal for which the users' perception of a company in the mining sector on the quality and use of GIS. For this study, we used a qualitative descriptive approach and, as a method, field research. The universe was composed of the active organizations that use GIS region Itabira / MG. The sample comprised eight employees of an organization who are users of a GIS and the criterion chosen was non-probability sampling accessibility. Data collection occurred through semi-structured interviews and processing of data by content analysis. Showed up, according to the questionnaires, Arcview software has a user friendly integration with other enterprise software and training are essential for users. The information must be constantly updated so there is no risk in working with obsolete data, ensuring that the database is always up to date, reliable and centralized. The positive features of the system were described by users, which gave various and considered as vital to the utilization and efficiency of the software and its results. The negative features of the system were described by users, which also list some critical points, which influence the results generated by the use of GIS. According to the general objective of this monograph, which was to analyze the users' perception of a mining company about the use of a GIS, we conclude that the users know the GIS mining company researched and considered as an essential tool for the environment in which processes are employed. It was proved that GIS has many advantages, like reliable database and full, that make clear its importance in the studied environment and its contribution to the decisions and actions taken are based on solid reliable and solid information.
\end{abstract}

Keywords: Geographic Information Systems. Perception. Quality. Mining sector company.

\title{
INTRODUÇÃO
}

A representação sobre mapas pode ser considerada como uma das práticas mais antigas exercida pela humanidade. O fato é atribuído à necessidade dos homens em representar o espaço real em seu correlativo desenho material, que lhes permitissem o uso dos mapas como forma de comparações, conhecimento, dados, leitura, interpretação, proporcionando uma estruturação e visão abrangente sobre um determinado lugar ou tema.

Sabe-se que uma empresa de mineração sobrevive do direito sobre recursos minerais, bem como compreende sua função na comercialização de minério de ferro, desempenhando papel importante para a sociedade no tocante à geração de emprego e renda e, principalmente, buscando atender às demandas dos clientes e consumidores.

Conseguir atender a todas as partes envolvidas e garantir um fornecimento eficaz de produtos aos seus clientes, muitas vezes, se torna uma tarefa complicada, o que faz com que as empresas mineradoras necessitem de novas áreas e/ou recursos para um atendimento adequado. Nesse sentido, para assegurar o mercado no fornecimento de minérios, se faz imprescindível não apenas o acompanhamento dos eventos ocasionados na produção como também o monitoramento dos diferentes dados relacionados a esse mercado minerador.

Com a necessidade de se elaborar condições cada vez mais ágeis e confiantes para o cumprimento dos trabalhos, [6] afirma que a utilização dos Sistemas de Informações Geográficas (SIG) apresenta-se como um recurso para o crescimento das empresas, apoiando- 
as na execução, no planejamento e no desenvolvimento de suas atividades, para que dessas informações possa ser obtido embasamento para decisões indispensáveis a uma gestão eficaz.

Ainda de acordo com [6], o aproveitamento dos SIG tem a seu favor a sistemática dos dados, tornando-os mais fáceis de entender, acessar e utilizar. Esse proveito pode ser inteiramente capitalizado pela empresa, seja diretamente, como ganho de capital, ou indiretamente, sob a forma de desenvolvimento na qualidade de seus serviços ou de aumento da produção. Assim, compreende-se que os benefícios do uso dos recursos dos sistemas de informações geográficas acrescentam ganhos e aumentam a competitividade para as empresas que utilizam estas ferramentas.

Esta pesquisa tem como objetivo analisar a percepção dos usuários de uma empresa do ramo de mineração acerca de uma solução de software do tipo SIG, do qual eles são manipuladores e possuem informações sobre seu funcionamento.

\section{SISTEMAS DE APOIO ÀS OPERAÇÕES}

Sabe-se que, em um ambiente empresarial, a informação é fundamental. Estratégias de informação contribuem para que as empresas se adaptem às mudanças e auxiliem na tomada de decisão. Portanto, se a informação for bem trabalhada e disseminada, assessoram as empresas no ganho de produtividade, melhor qualidade, redução de custos e qualidade para os clientes [15].

Por essa razão, de acordo com [15], inúmeras empresas perceberam que a informação é um recurso econômico e, diante disso, começaram a pensar em maneiras para melhor tratar esse novo bem. Com o objetivo de aumentar a eficiência e a eficácia, as empresas passaram a utilizar computadores, primordiais para o tratamento das informações e, com isso, criaram os Sistemas de Informação.

Para [17], os sistemas de informação têm por objetivo aumentar o acesso às informações, tornando mais simples a análise de grandes volumes de dados, o que auxilia a consolidação dos mesmos, que são valiosos e úteis para os usuários finais. Diante disso, cada vez mais as pessoas e as empresas necessitam de informações concisas e de qualidades para tomada de decisões. Assim, uma empresa necessita dispor de informações adequadas em termo de credibilidade, confiabilidade, uso e valor de obtenção.

De acordo com [22], um sistema de informação coleta, processa, armazena, analisa e dissemina informações com um propósito específico. Um sistema de informação abrange entradas e saídas, processa essas entradas e gera saídas que são enviadas para o usuário ou outros sistemas.

Segundo [21], a entrada em um sistema de informação é responsável pela captação de dados brutos de dentro da organização ou de seu ambiente externo. O processamento converte os dados obtidos na entrada em forma benéfica e adequada e, com isso, eles são classificados, analisados, manipulados e transformados para que a saída transfira a informação apropriada às pessoas ou às atividades nas quais a usarão. E, por último, há a realimentação ou feedback, que consiste em retornar a entrada de dados que serão refinados ou corrigidos, procedimento este fundamental para o sucesso da operação de um sistema.

Para que esse ciclo funcione adequadamente, os sistemas de informação contam com cinco recursos principais, conforme observado na Figura 1 a seguir. 


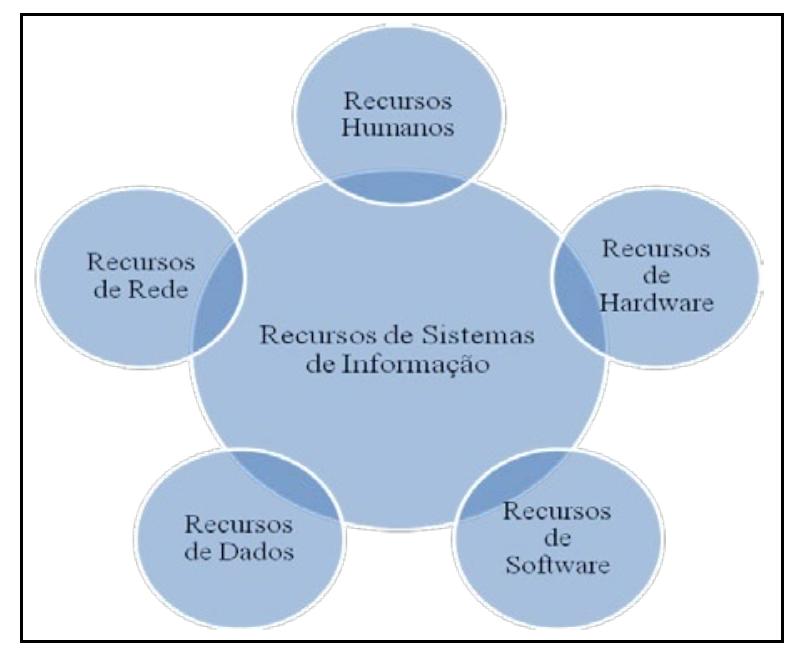

FIGURA 1 - Recursos de sistema de informação

Fonte: Adaptado de [17].

Os sistemas de informação de apoio às operações são voltados para processar dados gerados e usados por operações. Esses dados são usados pelos envolvidos no processo, porém não possuem uma formatação adequada para serem apresentados a gerentes, o que fica a cargo de um tratamento posterior em sistemas de informação gerencial.

Os sistemas de informação de apoio às operações, segundo [17], são divididos em: sistemas de processamento de transações, sistemas de controle de processos e sistemas colaborativos. O primeiro é responsável pelo processamento de dados obtidos em transações da empresa, existindo duas maneiras para que isto possa ser feito: em lote e em tempo real, onde a diferença está na forma de processamento das informações. Em lote, os dados são acumulados para posteriormente serem processados e, em tempo real, os dados são processados online.

\section{OS SITEMAS DE INFORMAÇÃO GEOGRÁFICA}

De acordo com [10], surgiram no Canadá na década de 60 os primeiros Sistemas de Informações Geográficas (SIG). O surgimento partiu da necessidade do governo em realizar um trabalho de levantamento dos recursos naturais. Porém, naquela época, os computadores eram muito caros e não existiam monitores gráficos de alta resolução, o que dificultava a criação de um software comercial genérico. Com isso, quem se interessava pelo sistema, encontrava muita dificuldade em usá-lo.

Na década de 80 houve a evolução dos hardwares, tornaram-se mais baratos os computadores pessoais que começaram a ser vendidos em grande escala, o que fez com que o SIG começasse a ser mais difundido e conhecido. Mas, foi no início da década de 90 que os SIG tomaram forças, prevalecendo seu aumento e uma crescente integração de seus usuários, facilitando, assim, o seu uso em aplicativos.

Existem diferentes e inúmeras definições de SIG nas literaturas, dentre as quais destacam-se algumas mais significativas. [3] explica que o SIG auxilia na exibição de dados espaciais referenciados, através de uma variedade de ferramentas que auxiliam ao reunir, guardar, restaurar, modificar e apresentar estes dados. Para [11], os SIG permitem a apresentação de mapas com informações gerais.

O principal motivo que leva a diversificação nos conceitos é o fato de o SIG ser uma união de várias áreas de conhecimento envolvidas, tais como: computação, equipamentos, empresas, banco de dados, mapas, dentre outras. Segundo [16], isso permite que cada área busque conceituar o SIG de acordo com seu campo de atuação e adequação.

Na definição de SIG relacionada aos equipamentos, destaca-se a visão de [16], onde 
esse sistema conta com dispositivos e programas computacionais para inserir e gerar mapas, além de contar também com os sistemas de comunicação necessários para ligar esses elementos, como as redes.

Para [19], existe um grande número de Sistemas de Informação Geográfica, cada um com características especificas em diferentes termos de tipos de elementos. Devido a estas dessemelhanças, existem alguns módulos presentes na maioria deles com a função de caracterizá-los. Esses módulos podem ser identificados na Figura 2.

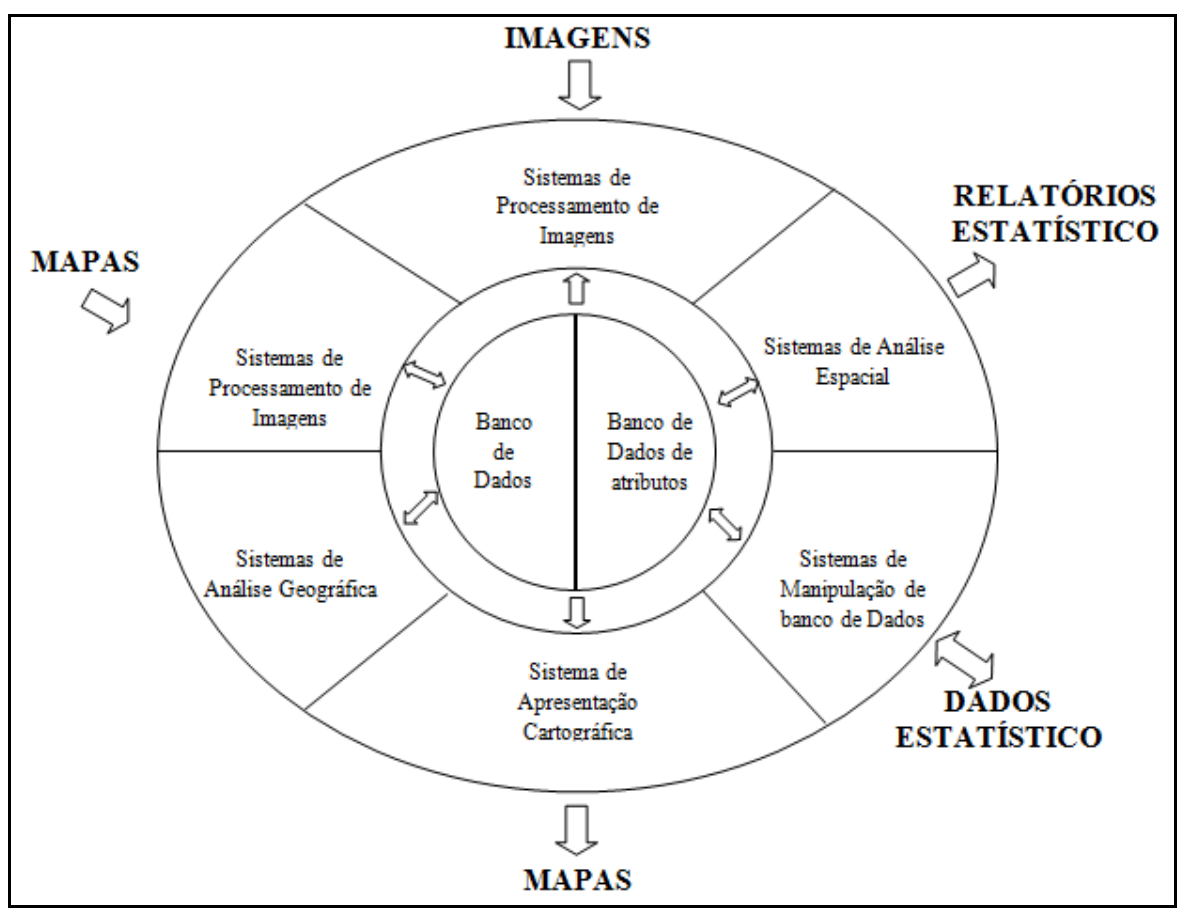

FIGURA 2 - Características de um SIG

Fonte: Adaptado de [19].

Segundo [20], a criação de mapas desenhados em papel, até pouco tempo, dificultava a análise das informações existentes como um todo. Atualmente, com o surgimento das tecnologias e o uso da informática a partir da década de 70, deu-se a origem do termo processamento, que faz uso de técnicas matemáticas e computacionais para tratar as informações geográficas no ambiente computacional.

De acordo com [20], o geoprocessamento vem sendo utilizado por diversas áreas como cartografia, análise de recursos naturais, transportes, comunicações, energia, planejamento urbano e mineração. As ferramentas de geoprocessamento permitem fazer análises complexas, a partir do momento que interagem informações de diversas fontes, contribuindo para a tomada de decisão dentro das empresas.

Os dados, ainda segundo [20], são mapas analógicos ou digitais, imagens de satélite e de radar, fotos aéreas, levantamentos topográficos e dados alfanuméricos. São armazenados em banco de dados e passam pelo processo de tratamento e análise usando métodos de modelagem de dados, geoestatística, aritmética lógica, análise de redes, análise topológica e reclassificação.

A partir de então, os dados estão prontos para serem usados em sistemas de informação como GIS (Geographic Information System), LIS (Land Information System), AM/FM (Automated Maping and Facilities Management), CAD (Computer Aided Design).

Conforme [16], atualmente existe dois tipos de SIG, os acadêmicos e os comerciais. Os comerciais são mais caros e demandam mais tempo de aprendizado, no mínimo seis meses, e são recomendados para trabalhos com grandes projetos, ou seja, grande volume de 
informações. Já os acadêmicos muitas vezes são gratuitos, o tempo de aprendizado é menor, são geralmente usados nas universidades e diversas instituições, podendo estar disponíveis em sites.

É imprescindível ressaltar a importância do SIG na mineração. De acordo com [5], o uso dos SIG em uma mineração é extenso, proporcionando inúmeros benefícios, conforme citado no quadro 1:

\section{QUADRO 1}

Aplicação do SIG na Mineração

\begin{tabular}{|c|c|}
\hline Banco de dados & $\begin{array}{l}\text { Cartografia; } \\
\text { Geológica; } \\
\text { Topografia; } \\
\text { Imagens de satélite; } \\
\text { Infraestrutura. } \\
\end{array}$ \\
\hline Pesquisa mineral & $\begin{array}{l}\text { Planejamento; } \\
\text { Integração; } \\
\text { Análise espacial avançada; } \\
\text { Mapeamento geológico; } \\
\text { Modelagem de depósitos. }\end{array}$ \\
\hline Geotecnia/hidrogeologia & $\begin{array}{l}\text { Gerenciamento de barragens; } \\
\text { Poços e modelagem 3D. }\end{array}$ \\
\hline Gestão ambiental & $\begin{array}{l}\text { Gerenciamento de risco; } \\
\text { Sistemas de emergência. }\end{array}$ \\
\hline Logística & $\begin{array}{l}\text { Rotas; } \\
\text { Sinalização; } \\
\text { Manutenção. }\end{array}$ \\
\hline Gestão de infraestrutura de suporte & $\begin{array}{l}\text { Energia; } \\
\text { Água; } \\
\text { Saneamento; } \\
\text { Telecomunicações. }\end{array}$ \\
\hline Suporte a projetos de engenharia & Instalações. \\
\hline
\end{tabular}

Fonte: Adaptado de [5].

\section{ANÁLISE DA UTILIZAÇÃO DO SIG EM UMA MINERADORA}

Após conceituar Sistemas de Informação e Sistemas de Informações Geográficas, descrever os conceitos, tipos e principais aplicações, além de citar o geoprocessamento, neste capítulo são apresentados os resultados obtidos a partir das entrevistas realizadas com a amostra escolhida para este estudo.

Este estudo utilizou uma abordagem qualitativa que, segundo [1], é aquela cujo pesquisador obtém suas informações a partir de coleta de dados em fenômenos pesquisados. O tipo utilizado foi o de pesquisa descritiva, no qual o fenômeno ou processo se relaciona com as variáveis ou fatores [13]. Para a coleta de dados foi utilizada a estratégia de entrevistas semiestruturadas, sendo considerada como um instrumento apropriado para a coleta de informações e interações com o campo [13].

O software utilizado na empresa estudada, que desempenha o papel do SIG naquele ambiente, é o ArcView. A pesquisa foi realizada com oito entrevistados, no período de 01/07/2013 à 31/07/2013. As informações colhidas a partir das respostas estão apresentadas a seguir.

Buscou-se saber dos entrevistados se receberam algum treinamento específico para manuseio do programa SIG, no caso o software ArcView, adotado pela empresa de mineração pesquisada. Buscou-se saber também como a experiência é adquirida no dia-a-dia, e qual a 
contribuição do treinamento para o perfeito funcionamento do sistema.

Todos os entrevistados afirmaram que realizaram treinamentos para manipulação do software adotado pela empresa. Afirmaram também que vão se aperfeiçoando no dia-a-dia, mas é importante que ocorra a reciclagem, pois o software pode ser atualizado em suas versões e isso poderia impactar no trabalho.

Foi possível verificar que as respostas dos entrevistados confirmam o que fala [4] sobre a importância da capacitação para os profissionais manipuladores da ferramenta, pois estes são os responsáveis pelo sucesso da implantação.

Quando questionados sobre os possíveis riscos/resultados decorrentes da utilização de dados desatualizados, como por exemplo, o uso de informações locais e não do servidor, todos entrevistados mostraram-se preocupados com esse quesito. Manipular informações erradas ou desatualizadas impacta drasticamente no resultado final das análises e tomada de decisões.

De acordo com os entrevistados, a manipulação de dados desatualizados se torna um risco eminente, gerando descréditos perante os usuários e comprometendo a tomada de decisão, o que pode trazer problemas para a empresa. Segundo [20], os dados armazenados no banco de dados carecem de proteção e acessos não autorizados, além de destruição, alteração intencional ou inclusão. Já [14] consideram o mau uso dos dados como intencional e acidental.

Sobre os possíveis riscos decorrentes da utilização de dados desatualizados, ressaltase o comentário do entrevistado 2 de que as informações podem ser discrepantes, situação a qual gera perdas acidentais que podem ser exemplificadas como a distribuição de inúmeros dados distribuídos em vários computadores, gerando diferentes anormalidades. A forma intencional está ligada diretamente a modificação, destruição, erros e não autorização de dados.

Os usuários foram questionados se conseguiriam imaginar como seriam os processos caso não fosse utilizado um SIG. Dentre os usuários, sete afirmaram a dificuldade e barreiras existentes, como, por exemplo, informações desatualizadas e inconfiabilidade nas informações. O processo chegou a ser comparado como os trabalhos realizados nos anos 80 . Já a minoria, representando por apenas 1 usuário da amostra, desconhece o processo sem o uso do SIG.

Todas as respostas obtdas, vão ao encontro da visão de [4], que complementa com a afirmação de que a implantação dessa tecnologia proporciona um grande auxílio aos trabalhos. $\mathrm{O}$ autor acrescenta ainda que o SIG é uma poderosa ferramenta gerencial de suporte à tomada de decisão da empresa. Assim, torna-se inviável o não uso desse software.

Foi questionado aos usuários que expressassem em quais pontos o SIG utilizado precisa ser melhorado para oferecer mais apoio e resultados melhores à empresa. As respostas apresentadas foram balanceadas, conforme informações do gráfico 1. 


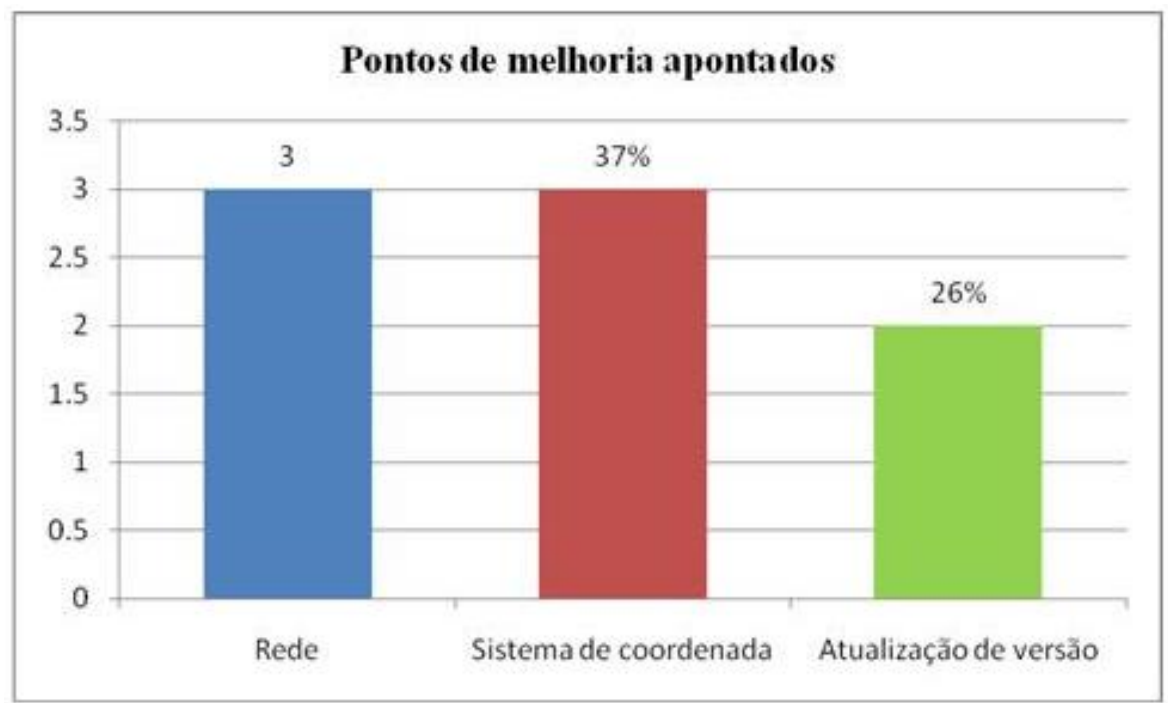

GRÁFICO 1 - Pontos de melhoria apontados pelos usuários

Fonte: Elaborado pelo usuário

Percebe-se a partir da pesquisa, que um dos pontos em que o SIG poderia ser melhorado seria a rede atender adequadamente às necessidades dos usuários. Informações semelhantes são encontradas em [7] que afirmam que um exemplo dos sistemas comerciais que são baseados numa estratégia dual é o ArcView. Nele, uma desvantagem é que as consultas são mais lentas, pois são processadas separadamente. A parte convencional da consulta é processada pelo SGBD separado da parte espacial, que é processada pelo aplicativo utilizando os arquivos proprietários [7].

Relacionando as questões obtidas através da pesquisa e as condições apresentadas pelos autores acima citados, nota-se que a lentidão da rede é normal, visto que o software ArcView apresenta essa desvantagem.

Os usuários foram questionados quanto à segurança e capacidade do SIG em proteger suas informações e fornecê-las apenas às pessoas autorizadas. Todos os usuários disseram do conforto das informações serem liberadas apenas para os autorizados e administradores do software.

O acesso aos dados é controlado de acordo com os direitos definidos, além dos níveis de autorização e controle de acesso de cada usuário, a segurança de quem é responsável por esse gerenciamento. No que diz respeito à segurança, [23] advoga que a segurança de um software tem a capacidade de avaliar tanto o nível em que os dados são protegidos de acesso não autorizados, como também o nível em que as informações são disponibilizadas por usuários autorizados.

Com as respostas obtidas, conforme Figura 3, foi possível observar o contentamento dos usuários em trabalhar com dados seguros, com informações sendo liberadas apenas para usuários autorizados e administradores do software, o que confere maior confiabilidade ao mesmo. 


\section{Importância de capacitação profissional para os usuários}

O soffware Archiew possui uma interação amigável

Banco de dados atualizado, confiável e centralizado

Risco em trabalhar com informaçôes desatualizados

Inviabilidade da tomada de decisão sem o uso do SIG

Figura 3: Análise do primeiro objetivo específico

Fonte: Dados da pesquisa

De acordo com os entrevistados, entende-se que os resultados do software ArcView são confiáveis, padronizados, os dados são centralizados, com a ressalva de que o banco de dados esteja sempre atualizado. Isso é confirmado em [8], o qual esclarece que um SIG integra um SGBD e simplifica alguns serviços para o programador, tais como: armazenar e recuperar dados, permitir acesso lógico independente da estrutura de armazenamento físico, permitir acesso a dados padronizados, definir restrições de consistência para serem automaticamente impostas, controlar acesso concorrente por múltiplos usuários e proteger contra perda ou acesso não autorizado.

Solicitou-se aos participantes que apontassem quais as principais vantagens do software SIG utilizado pela empresa. Todos usuários evidenciaram a resposta com exemplos bastante significativos, conforme mostrado no gráfico 2 .

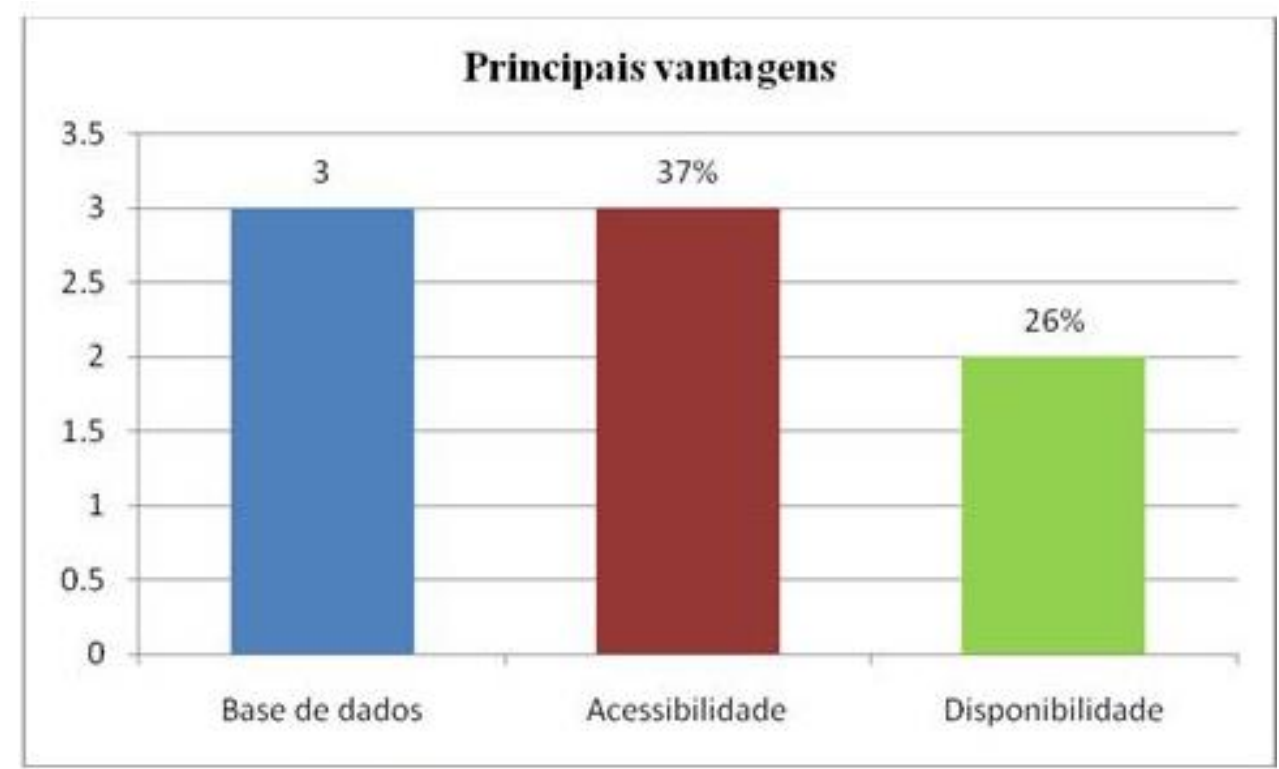

GRÁFICO 2 - Principais vantagens apontados pelos usuários

Fonte: Elaborado pelo usuário

A partir dos dados coletados, destaca-se o depoimento do entrevistado 2, ao afirmar que o SIG apresenta uma base de dados confiável e integrada. Tal colocação pode ser relacionada à afirmação de [4], que afirma que o sucesso de um SIG que faz o uso de banco de dados está na confiabilidade das informações, e isso é garantido quando se faz o uso de dados concisos, extremamente confiáveis. 
Quando questionados se o SIG oferece alguma vantagem para os gerentes analisarem os resultados, facilitando assim a tomada de decisão, as respostas foram unânimes de que o software permite uma análise crítica do projeto, a rapidez e a confiabilidade das informações, além da visualização rápida e de fácil entendimento.

Essa realidade é mostrada por [9], o qual concebe o SIG como um sistema de suporte à decisão que integra dados referenciados espacialmente num ambiente de respostas a problemas.

O envolvimento de todas as áreas abrangidas numa organização é fundamental para que haja o sucesso da mesma. Conforme evidenciado pelos usuários, o software permite uma boa integração entre as áreas. Segundo [4], com a aplicação do SIG, é possível a obtenção de um banco de dados único que pode ser compartilhado por várias áreas ou departamentos. Para a mesma autora, a utilização de um SIG na mineração é de grande importância, pois oferece informações de inúmeras aplicações envolvidas.

Diante dos dados analisados, conforme Figura 4 pode-se perceber que o software avaliado apresenta impactos positivos no ambiente organizacional, como: confiabilidade nas informações, agilidade na tomada de decisões e banco de dados integrado, pois ele trabalha com dados sempre atualizados e, com isso, proporciona confiabilidade e eficiência no processo, auxiliando a todos e, em especial, os gerentes na tomada de decisão.

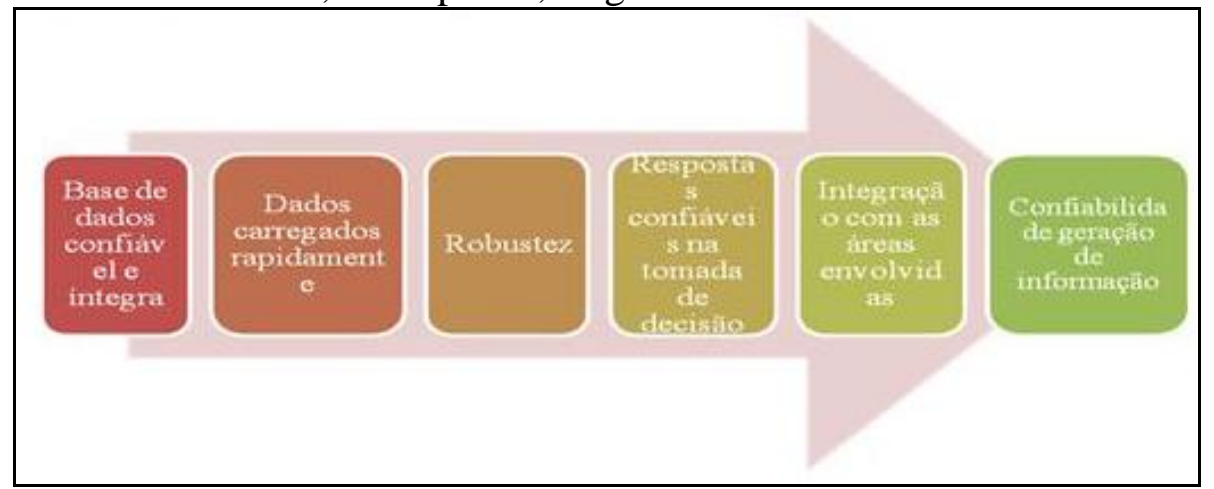

Figura 4: Análise do segundo objetivo específico

Fonte: Dados da pesquisa

Os usuários foram questionados a respeito das possíveis características negativas relacionadas ao SIG adotado pela empresa. Dentre os respondentes, a maioria dos usuários apontou a restrição de licenças como um dos pontos negativos do SIG.

De acordo com a maioria dos entrevistados, existe expectativa de que os problemas ainda existentes, como a restrição do uso das licenças impossibilitando a disseminação do sistema, sejam resolvidos. Conforme [2], o termo de licença de software concebe o direito e as regras de utilização de um determinado software instalado em um computador.

Tratando-se de alguns aspectos em relação à performance, sobre o que poderia ser melhorado, seis dos usuários opinaram que a rede seria "o grande gargalo" a ser aperfeiçoado.

Um sistema deve ser ágil e eficaz. Sistemas e redes de computadores devem ser desenvolvidos de forma a gerar um atendimento eficiente, permitindo aos usuários pleno atendimento. Essa realidade é mostrada por [18], que afirma que a dispersão da informação espacial em ampla escala é um dos maiores desafios científicos e tecnológicos existentes.

Procurou-se conhecer também qual aspecto o usuário vê como a pior característica do SIG. A maioria dos entrevistados citou a rede, e a minoria citou as informações pesadas que o SIG origina.

Ainda sobre os aspectos que impactam negativamente na eficácia do SIG, os usuários foram questionados sobre quais as principais desvantagens encontradas atualmente, decorrentes da forma de utilização do SIG. A maioria dos usuários mencionou a lentidão da rede e velocidade de processamento, alguns citaram a falta de treinamento e outros afirmaram 
desconhecer desvantagens.

Percebe-se que a rede de comunicação da empresa e a velocidade de processamento provocam inquietação nos entrevistados. Tais colocações estão de acordo com [12], que afirma que, dependendo da velocidade de acesso, essa estratégia pode resultar em longos e sucessivos períodos de espera.

Diante das desvantagens apresentadas, acredita-se que o descontentamento dos usuários do SIG seria a rede como ponto a ser melhorado, visto que ela é responsável pela lentidão no processamento das informações.

Verificou-se, através da análise, os aspectos que impactam negativamente na produtividade em um SIG numa empresa de mineração, conforme Figura 5, de acordo com os pontos verificados pelos usuários que apontaram: restrição de licenças pelo fato de cada usuário possuir uma licença em sua maquina, com isto restringindo a disseminação do SIG; lentidão da rede afetando o processamento das imagens e causando uma imprecisão de trabalhar com dados advindos de fontes erradas; desconhecimento por parte de um usuário; informações pesadas que o SIG origina; a falta de treinamento e máquinas incompatíveis. É preciso chamar a atenção para a lentidão do software, que se mostrou recorrente entre as colocações negativas.

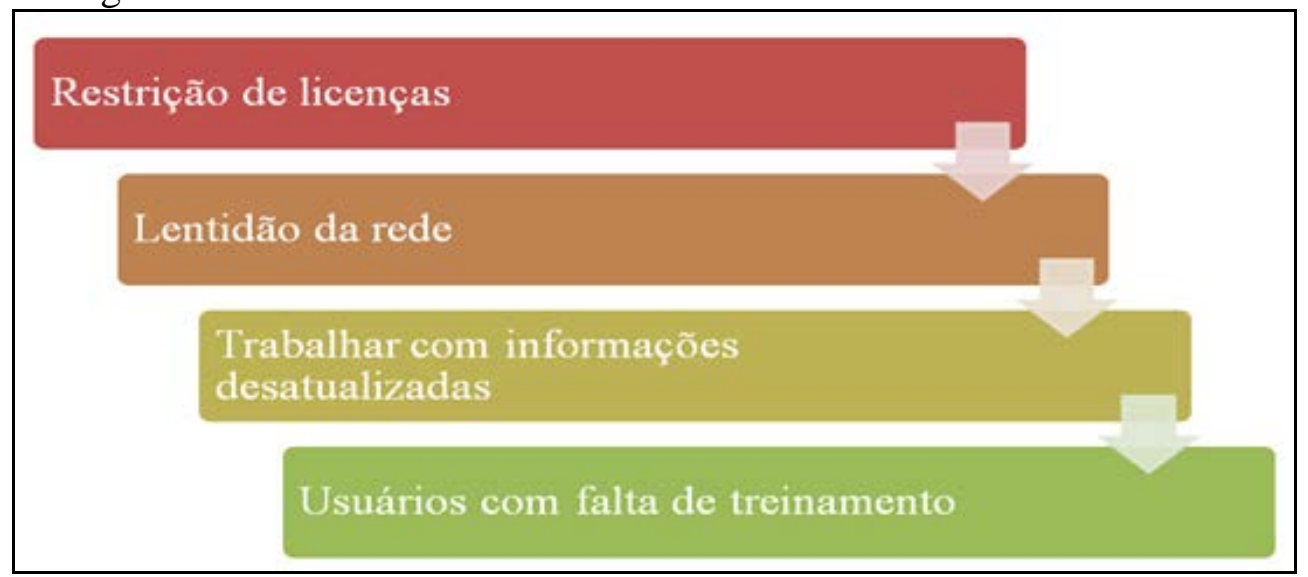

Figura 5: Análise do terceiro objetivo específico

Fonte: Dados da pesquisa

\section{CONCLUSÕES}

Evidenciou-se que, de acordo com as entrevistas, o software Arcview possui uma integração amigável com os demais softwares da empresa. Porém, para que isso aconteça, é fundamental que os usuários sejam capacitados através de treinamentos.

As características positivas do software foram descritas pelos usuários, que apontaram várias vantagens como: base de dados confiável e íntegra, de fácil acessibilidade e disponibilidade de acesso; os dados são carregados rapidamente; robustez pelo fato de processar inúmeras informações; a disponibilização das informações são rápidas, o que leva satisfação para os usuários ao oferecer respostas confiáveis e facilidade de geração de informações. É notório aos usuários que o software é vantajoso e atrativo, pois corrobora para a rapidez na tomada de decisão e montagem de inúmeros cenários, além de confiabilidade e padronização dos dados.

As características negativas do software foram descritas pelos usuários, que listaram alguns pontos como: restrição de licenças pelo fato de cada usuário possuir a sua licença em sua máquina; a lentidão da rede da empresa foi observada, impactando no processamento das imagens; o risco em trabalhar-se com informações erradas; informações pesadas que o SIG origina.

Pode-se concluir que os usuários conhecem o sistema de informações geográficas da 
empresa de mineração pesquisada, onde há grande satisfação dos usuários para com o sistema. Comprovou-se que o SIG apresenta características positivas, como: base de dados confiável e íntegra, características negativas como: restrição de licenças, lentidão na rede e dentre outras já citadas, características essas que foram enumeradas pelos estudiosos do tema.

\section{REFERÊNCIAS BIBLIOGRÁFICAS}

[1] APPOLINÁRIO, Fabio. Metodologia da Ciência: filosofia e prática da pesquisa. São Paulo: Cengage Learning, 2009.

[2] BEZERRA, Tárcio R. Bouncer - Uma Solução Distribuída para Controle de Licenças de Software, Dissertação de Mestrado, COPIN/DSC/UFPB - Campus II, dezembro de 1996.

[3] BURROUGH, P. A. Principles of Geographical Information Systems: Methods and Requirements for Lauduse Planning. Clarendon: Oxford. 1986.

[4] CALAZANS, P.M.P. Geoprocessamento Aplicado à Pesquisa Mineral. 2000. 31p. Monografia (Especialização em Geoprocessamento) - UFMG, Belo Horizonte. 2000. Disponível em: <http://dominiopublico.gov.br>. Acesso em: 02 fev. 2014.

[5] CALAZANS, P.M.P.; DOMINGUES, A. L. Implantação e funcionalidades de um sistema de informação geográfica mineral na Companhia Vale do Rio Doce - CVRD. In: XIII Simpósio Brasileiro de Sensoriamento Remoto, 21-26 abril, 2007. Anais... Florianópolis, INPE, p. 1979-1986.

[6] CÂMARA, Gilberto. Sistemas de Informação geográfica para aplicações ambientais e cadastrais: Uma visão Geral. Instituto Nacional de Pesquisas Espaciais - INPE, 1999. Disponível em: <www.dpi.inpe.br/geopro/trabalhos/analise.pdf>. Acesso em: 03 mar. 2013.

[7] CÂMARA, G. ; QUEIROZ, G. R. Arquitetura de Sistemas de Informação Geográfica. São José dos Campos, INPE, 2004.

[8] CÂMARA Neto, G. Modelos, linguagens e arquiteturas para banco de dados geográficos. (Preliminar da Tese de Doutorado). Instituto Nacional de Pesquisas Espaciais, 1995.

[9] COWEN, D. J. GIS versus CAD versus DBMS: what are the differences, Photogrammetric Engineering and Remote Sensing, 54 (11):1551-1554, 1988.

[10] DAVIS, Clodoveu; FONSECA, Frederico. Introdução aos Sistemas de Informações Geográficas. Belo Horizonte: Prodabel, 2001.

[11] DEVINE, H. A. e FIELD, R. C.The Gist of GIS. Journal of Forestry. August: 1986.

[12] ESRI Environmental Systems Research Institute Inc. Object Modelling and Geodatabases. ArcInfo 8.0 Pre-Release: 2000.

[13] JUNG, Carlos Fernando. Metodologia para Pesquisa \& Desenvolvimento. Rio de Janeiro: Axcel Books do Brasil, 2004.

[14] KORTH, Henry F.; SILBERSCHATZ Abraham. Sistema de Banco de Dados. 2. ed. São Paulo: Mcgraw-hill, 1994.

[15] MCGEE, James; PRUSAK Laurence. Gerenciamento estratégico da Informação: aumente a competitividade e eficiência de sua empresa utilizando a informação como uma ferramenta estratégica. Rio de Janeiro: Elsevier, 1994. 
[16] MIRANDA, José Iguelmar. Fundamentos de Sistemas de Informação Geográficas. 1. ed. Brasília: Embrapa Informação Tecnológica, 2005.

[17] O’BRIEN, James A. Sistemas de Informação e as decisões gerenciais na era da internet. 2. ed. São Paulo: Saraiva, 2004.

[18] OSSES, J. R. Arquiteturas cliente-servidor para disseminação de dados geográficos. (Dissertação de Mestrado do Curso de Computação Aplicada) - Instituto Nacional de Pesquisas Espaciais, São José dos Campos, 2000, Disponível em:

<http://www.dpi.inpe.br/teses/jroberto/tesejro.pdf> Acesso em 31 ago. 2013.

[19] ROCHA, Cézar Henrique Barra. Geoprocessamento: Tecnologia Transdisciplinar, Juiz de Fora, MG: Ed. Do Autor, 2000.

[20] SILVA, Reginaldo Macedônio da. Introdução ao Geoprocessamento: Conceitos, técnicas e aplicações. Novo Hamburgo: Feevale, 2007.

[21] STAIR, Ralph M.. Princípios de sistemas de informação: Uma abordagem gerencial. 2.ed.São Paulo: LTC, 1998.

[22] TURBAN, Efraim; R. Kelly Rainer Jr.; Richard E. Plotter. Administração de tecnologia da informação. Rio de Janeiro: Elsevier, 2003.

[23] WAZLAWICK, Raul Sidnei. Engenharia de software: conceitos e práticas. Rio de Janeiro: Elsevier, 2013. 